BMJ Open

Diabetes

Research

\& Care

\title{
Elevated serum adiponectin, elevated tumor necrosis factor- $\alpha$ and decreased transthyretin in Japanese elderly women with low grip strength and preserved insulin sensitivity
}

\author{
Mika Takeuchi, ${ }^{1}$ Ayaka Tsuboi, ${ }^{2,3}$ Satomi Minato, ${ }^{2,4}$ Megumu Yano, ${ }^{2}$ \\ Kaori Kitaoka, ${ }^{2,5}$ Miki Kurata, ${ }^{1,2}$ Tsutomu Kazumi, ${ }^{\oplus, 6}$ Keisuke Fukuo $^{1,2}$
}

To cite: Takeuchi M, Tsuboi A, Minato S, et al. Elevated serum adiponectin, elevated tumor necrosis factor- $\alpha$ and decreased transthyretin in Japanese elderly women with low grip strength and preserved insulin sensitivity. BMJ Open Diab Res Care 2019;7:e000675. doi:10.1136/ bmjdrc-2019-000675

- Additional material is published online only. To view please visit the journal online (http://dx.doi.org/10.1136/ bmjdrc-2019-000675)

Received 12 April 2019 Revised 30 May 2019 Accepted 1 July 2019
Check for updates

(c) Author(s) (or their employer(s)) 2019. Re-use permitted under CC BY-NC. No commercial re-use. See rights and permissions. Published by BMJ.

For numbered affiliations see end of article.

Correspondence to Dr Tsutomu Kazumi; kazumi@mukogawa-u.ac.jp

\section{ABSTRACT}

Objective To determine if serum adiponectin levels are associated with low grip strength and/or low muscle mass in the elderly independently of insulin resistance and inflammation.

Research design and methods Cross-sectional associations were analyzed by logistic regression between low grip strength and/or low muscle mass and body composition, elevated serum adiponectin ( $\geq 20 \mathrm{mg} / \mathrm{L})$, and biomarkers of nutrition status, insulin resistance and inflammation in 176 community-living Japanese elderly women. Sarcopenia was evaluated using the Asian criteria. Results In bivariate analyses, low grip strength $(n=71)$ was positively associated with age, log tumor necrosis factor- $\alpha$ and hyperadiponectinemia $(n=34)$ and inversely with body weight, height, skeletal muscle mass, serum albumin, transthyretin (TTR), fat mass, serum zinc and hemoglobin (all $\mathrm{p}<0.01$ ). In a fully adjusted model, TTR (OR 0.90; $95 \% \mathrm{Cl} 0.83$ to $0.98, p=0.01$ ) in addition to age $(p=0.01)$, height $(p<0.001)$ and skeletal muscle mass $(p=0.018)$ emerged as independent determinants of low grip strength. Mean waist circumference and high-density lipoprotein cholesterol did not differ between women with and without low grip strength and were within the respective normal range. Although hyperadiponectinemia was a single metabolic correlate of low muscle mass $(\mathrm{n}=87)$, low serum cholesterol, albumin and TTR in addition to hyperadiponectinemia were associated with sarcopenia $(n=45)$.

Conclusions Hyperadiponectinemia, elevated tumor necrosis factor-alpha and decreased TTR, a biomarker of age-related catabolic states, were found in community-living Japanese elderly women with low grip strength and preserved insulin sensitivity although hyperadiponectinemia was a single correlate of low muscle mass.

\section{INTRODUCTION}

Sarcopenia, age-related declines in muscle mass, strength and function, is often an important antecedent of the onset of disability in older adults. ${ }^{12}$ The mechanisms of sarcopenia remain unclear. Muscle function is

\section{Significance of this study}

What is already known about this subject?

- Low muscle strength and mass in the elderly have been reported in separate studies to be associated with nutrition, insulin resistance, inflammation and elevated serum adiponectin. However, studies which evaluated simultaneously all these conditions were limited.

What are the new findings?

- Low grip strength in community-living Japanese elderly women was associated with decreased transthyretin, a marker of nutrition and age-related catabolic states, elevated tumor necrosis factor- $\alpha$, an inflammatory cytokine, and elevated serum adiponectin, but not with insulin resistance although low muscle mass was associated with hyperadiponectinemia alone.

How might these results change the focus of research or clinical practice?

- Association of adiponectin with muscle strength and mass suggests an opportunity to identify those at high risk of this complication and to discover insights into the mechanisms of this very early adverse event.

influenced by lifestyle, biological and psychosocial factors. ${ }^{2}$ Lifestyle factors include physical inactivity and inadequate nutrition. Changes in endocrine function, mitochondrial dysfunction and insulin resistance (IR) are examples of biological factors. ${ }^{12}$ Cross-sectional studies have reported that IR, a key player in the development of type 2 diabetes, was associated with muscle strength in community-dwelling elderly people. ${ }^{3-5}$ Some but not all prospective studies have demonstrated that low muscle strength predicts incident type 2 diabetes. ${ }^{6-8}$ 
Another important biological factor for age-related sarcopenia is systemic chronic low-grade inflammation. ${ }^{1}$ In a clinical setting of inflammatory diseases, such as cancer and congestive heart failure, tumor necrosis factor-alpha (TNF- $\alpha$ ), produced by circulating mononuclear cells, is an important cytokine in muscle wasting and weakness. ${ }^{9} \mathrm{In}$ the general population, consistent associations have been reported between age-related chronic low-grade inflammation and sarcopenia. ${ }^{10}$

Serum adiponectin was elevated in malnourished, underweight female patients with anorexia nervosa and decreased after weight gain. ${ }^{11}$ The authors hypothesize that increased adiponectin levels may have a protective role in maintaining energy homeostasis during extreme malnourishment because the serum adiponectin is important to maintaining energy homeostasis under energy shortage conditions. ${ }^{12}$ Serum adiponectin increased with age and this was associated with decreasing weight. ${ }^{13}$ Higher serum adiponectin levels have been reported to be associated with muscle weakness in the general population and the elderly. ${ }^{13-16}$ We have recently demonstrated that adiponectin was elevated in elderly women with anemia and reduced renal function. ${ }^{17}$ Because studies are limited which evaluated a broad range of above-mentioned variables associated with sarcopenia and because women compared with men and elderly compared with younger people had lower muscle strength, we tested whether elevated adiponectin levels may be associated with low grip strength and low muscle mass, either alone or in combination, in elderly Japanese women and examined whether this association, if present, may be independent of IR, low-grade inflammation, nutrition status and comorbidities.

\section{METHODS}

Among 202 Japanese elderly women reported in our previous studies, ${ }^{18} 19176$ women (aged 50-96 years) who underwent bioelectrical impedance analysis and measurements of grip strength were cross-sectionally studied. They were residents in Nishinomiya, Hyogo, Japan and were recruited as volunteers by local welfare commissioners from the city of Nishinomiya. Because we assessed effects of subclinical, low-grade inflammation, subjects who reported that they were under treatment for acute or chronic inflammatory diseases, cancer, cardiovascular, hepatic and renal diseases were excluded from the study. The study was in accordance with the Helsinki declaration. There was no significant difference in anthropometric and biochemical characteristics between 176 women studied in the present study and remaining 26 women without grip strength measurements (data not shown).

Anthropometric indices and grip strength were measured, and venous blood was drawn after breakfast between 09:30 and 11:30. Breakfast was not standardized and time interval between breakfast and blood samplings varied. Fat and lean mass were measured using bioelectrical impedance analysis (InBody 430, Biospace, Tokyo, Japan). Fat mass index (FMI, in $\mathrm{kg} / \mathrm{m}^{2}$ ) was calculated as fat mass in kilograms divided by height squared in meter. Appendicular skeletal muscle mass index (SMI, in $\mathrm{kg} / \mathrm{m}^{2}$ ) was calculated as appendicular skeletal muscle mass (SM) in kilograms divided by height squared in meter. $^{20}$

Grip strength was measured with a handheld dynamometer (TKK5401, Takei Scientific Instruments, Tokyo, Japan) as previously reported. ${ }^{18}$ Two trials for the dominant hand were performed and the stronger results were used in analyses. As participants were all women, low muscle mass was classified as a SMI $<5.7 \mathrm{~kg} / \mathrm{m}^{2}$ and low muscle strength was defined as a grip strength $<18 \mathrm{~kg} .{ }^{20}$ Sarcopenia was defined according to the recommended algorithm of the Asian Working Group for Sarcopenia. ${ }^{20}$

We evaluated routine laboratory parameters, including plasma glucose, insulin, serum lipids and lipoproteins, as previously reported. ${ }^{21}$ Serum levels of albumin, transthyretin (TTR), zinc, iron and copper were measured as previously reported. ${ }^{22}{ }^{23}$ Adiponectin was assayed by a sandwich ELISA (Otsuka Pharmaceutical Co., Ltd., Tokushima City, Japan). Intra-assay and interassay coefficient of variation (CV) were $3.3 \%$ and $7.5 \%$, respectively. Leptin was assessed by a RIA kit from LINCO research (St. Charles, Missouri, USA; interassay $\mathrm{CV}=4.9 \%$ ). High-sensitivity C-reactive protein (hsCRP) was measured by an immunoturbidometric assay with the use of reagents and calibrators from Dade Behring Marburg $\mathrm{GmbH}$ (Marburg, Germany; interassay CV $<5 \%$ ). TNF- $\alpha$ were measured by immunoassays (R\&D Systems, Minneapolis, Minnesota, USA; interassay CV $<6 \%$ ). Plasminogen activator-inhibitor-1 (PAI-1) was measured by an ELISA method (Mitsubishi Chemicals; interassay CV $<8 \%$ ). Complete blood cell count was analyzed using an automated blood cell counter (Sysmex XE-2100, Sysmex, Kobe, Japan). Anemia was defined as hemoglobin $<120 \mathrm{~g} / \mathrm{L}$ (WHO).

Serum creatinine was measured enzymatically using an autoanalyzer (AU 5200, Olympus, Tokyo, Japan). The estimated glomerular filtration rate (eGFR) was calculated using the equation recommended by the Japanese Society for Nephrology. ${ }^{24}$ Chronic kidney disease (CKD) G3b was defined as eGFR $<45 \mathrm{~mL} / \mathrm{min} / 1.73 \mathrm{~m}^{2} .^{25}$

Data were presented as mean $\pm \mathrm{SD}$ unless otherwise stated. Due to deviation from normal distribution hsCRP and TNF- $\alpha$ were logarithmically transformed for statistical analyses. Adiponectin was evaluated as a categorized valuable; hyperadiponectinemia $(\geq 20 \mathrm{mg} / \mathrm{L}){ }^{13}$ Differences between two groups were analyzed by t-test and frequencies of conditions by $\chi^{2}$ test. Associations of confounders with low grip strength, low SMI and sarcopenia were determined by bivariate and multivariate logistic regression analyses providing OR with 95\% CI. Dependent variables included in multivariate logistic regression analyses were variables that showed significant difference between two groups (the presence and the absence of low grip strength, low muscle mass or sarcopenia). A two-tailed $\mathrm{p}<0.05$ was considered statistically 
Table 1 Characteristics of elderly women stratified by the presence and absence of low grip strength

\begin{tabular}{|c|c|c|c|}
\hline & Low grip s & & \\
\hline & Yes & No & \\
\hline & $n=71$ & $\mathrm{n}=105$ & $P$ values \\
\hline Age (years) & $80.8 \pm 5.5$ & $74.0 \pm 8.5$ & $<0.01$ \\
\hline Height $(\mathrm{cm})$ & $145.4 \pm 5.3$ & $151.1 \pm 5.4$ & $<0.01$ \\
\hline Weight (kg) & $46.1 \pm 7.1$ & $52.2 \pm 7.1$ & $<0.01$ \\
\hline $\mathrm{BMI}\left(\mathrm{kg} / \mathrm{m}^{2}\right)$ & $21.8 \pm 3.2$ & $22.8 \pm 2.8$ & 0.031 \\
\hline $\mathrm{FMI}\left(\mathrm{kg} / \mathrm{m}^{2}\right)$ & $7.0 \pm 2.7$ & $7.6 \pm 2.2$ & 0.139 \\
\hline $\mathrm{SMI}\left(\mathrm{kg} / \mathrm{m}^{2}\right)$ & $5.4 \pm 0.6$ & $5.9 \pm 0.6$ & $<0.01$ \\
\hline Waist circumference $(\mathrm{cm})$ & $85.1 \pm 9.8$ & $87.3 \pm 8.8$ & 0.125 \\
\hline Fat mass $(\mathrm{kg})$ & $14.8 \pm 5.6$ & $17.2 \pm 5.0$ & 0.002 \\
\hline Skeletal muscle mass $(\mathrm{kg})$ & $11.5 \pm 1.5$ & $13.6 \pm 2.0$ & $<0.01$ \\
\hline Grip strength $(\mathrm{kg})$ & $15.0 \pm 2.6$ & $23.2 \pm 3.2$ & $<0.01$ \\
\hline PB glucose (mg/dL) & $101 \pm 38$ & $98 \pm 22$ & 0.456 \\
\hline PB insulin $(\mu \mathrm{U} / \mathrm{mL})$ & $9.4 \pm 9.1$ & $7.3 \pm 5.4$ & 0.161 \\
\hline PB triglycerides (mg/dL) & $136 n \pm 71$ & $140 \pm 82$ & 0.739 \\
\hline Cholesterol (mg/dL) & $214 \pm 28$ & $221 \pm 34$ & 0.128 \\
\hline HDL cholesterol (mg/dL) & $61 \pm 13$ & $65 \pm 15$ & 0.111 \\
\hline Leptin (ng/mL) & $7.3 \pm 5.0$ & $8.0 \pm 4.5$ & 0.307 \\
\hline Adiponectin (mg/L) & $16.4 \pm 9.5$ & $12.5 \pm 6.2$ & 0.003 \\
\hline Leucocytes $\left(10^{9} / \mathrm{L}\right)$ & $6.0 \pm 1.6$ & $6.2 \pm 1.6$ & 0.542 \\
\hline PAI-1 (ng/mL) & $24 \pm 10$ & $25 \pm 12$ & 0.337 \\
\hline hsCRP $(\mu \mathrm{g} / \mathrm{dL})$ & $83 \pm 101$ & $87 \pm 118$ & 0.814 \\
\hline TNF- $\alpha(p g / m L)$ & $1.8 \pm 0.9$ & $1.6 \pm 1.1$ & 0.025 \\
\hline Red blood cells $\left(10^{12} / \mathrm{L}\right)$ & $413 \pm 40$ & $429 \pm 31$ & 0.007 \\
\hline Hemoglobin (g/L) & $125 \pm 12$ & $130 \pm 10$ & 0.004 \\
\hline Hematocrit (\%) & $40.0 \pm 3.4$ & $41.3 \pm 3.2$ & 0.008 \\
\hline Albumin (g/dL) & $4.3 \pm 0.3$ & $4.5 \pm 0.2$ & $<0.01$ \\
\hline Iron $(\mu \mathrm{g} / \mathrm{dL})$ & $91 \pm 29$ & $95 \pm 27$ & 0.256 \\
\hline Copper $(\mu \mathrm{g} / \mathrm{dL})$ & $110 \pm 17$ & $109 \pm 14$ & 0.515 \\
\hline $\operatorname{Zinc}(\mu \mathrm{g} / \mathrm{dL})$ & $74 \pm 11$ & $79 \pm 11$ & 0.005 \\
\hline Transthyretin (mg/dL) & $25 \pm 5$ & $28 \pm 5$ & $<0.01$ \\
\hline Creatinine (mg/dL) & $0.69 \pm 0.18$ & $0.68 \pm 0.13$ & 0.698 \\
\hline eGFR $\left(\mathrm{mL} / \mathrm{min} / 1.73 \mathrm{~m}^{2}\right)$ & $64 \pm 13$ & $66 \pm 13$ & 0.356 \\
\hline Anemia (n, \%) & $19,26.8$ & $17,16.2$ & 0.088 \\
\hline CKD G3b (n, \%) & $8,11.3$ & $4,3.8$ & 0.069 \\
\hline Hyperadiponectinemia (n, \%) & $21,29.6$ & $13,12.4$ & 0.006 \\
\hline
\end{tabular}

Mean \pm SD or n, \%.

BMI, body mass index; CKD, chronic kidney disease; FMI, fat mass index; HDL, high-density lipoprotein; PAl-1, plasminogen activatorinhibitor-1; PB, post-breakfast; RBC, red blood cells; SMI, skeletal muscle mass index; TNF- $\alpha$, tumor necrosis factor-alpha; eGFR, estimated glomerular filtration rate; hsCRP, high-sensitivity C-reactive protein.

significant. All calculations were performed with SPSS system V.15.0.

\section{RESULTS}

Among 176 women, 71 women (40.3\%) had low grip strength (table 1 ) and 87 women $(49.4 \%)$ had low SMI (table 2). There were 45 women $(25.6 \%)$ with sarcopenia (table 3). Elderly women on average were of normal weight. Their insulin sensitivity appeared to be maintained as their mean values of waist circumference, BMI and HDL cholesterol were within respective normal range. Their nutrition status also appeared to be 
Table 2 Characteristics of women with low muscle mass

\begin{tabular}{|c|c|c|c|}
\hline & Low musc & & \\
\hline & Yes & No & \\
\hline & $\mathrm{n}=87$ & $\mathrm{n}=89$ & $P$ values \\
\hline Age (years) & $78.4 \pm 6.7$ & $75.1 \pm 9.1$ & 0.006 \\
\hline Height (cm) & $147.6 \pm 5.7$ & $150.0 \pm 6.1$ & 0.008 \\
\hline Weight (kg) & $45.4 \pm 5.9$ & $53.9 \pm 6.8$ & $<0.001$ \\
\hline $\operatorname{BMl}\left(\mathrm{kg} / \mathrm{m}^{2}\right)$ & $20.9 \pm 2.6$ & $24.0 \pm 2.6$ & $<0.001$ \\
\hline $\mathrm{FMI}\left(\mathrm{kg} / \mathrm{m}^{2}\right)$ & $6.6 \pm 2.3$ & $8.1 \pm 2.3$ & $<0.001$ \\
\hline $\mathrm{SMI}\left(\mathrm{kg} / \mathrm{m}^{2}\right)$ & $5.2 \pm 0.4$ & $6.2 \pm 0.5$ & $<0.001$ \\
\hline Waist circumference $(\mathrm{cm})$ & $83.9 \pm 9.5$ & $88.9 \pm 8.3$ & $<0.001$ \\
\hline Fat mass $(\mathrm{kg})$ & $14.3 \pm 5.0$ & $18.2 \pm 5.1$ & $<0.001$ \\
\hline Skeletal muscle mass $(\mathrm{kg})$ & $11.4 \pm 1.3$ & $14.1 \pm 1.8$ & $<0.001$ \\
\hline Grip strength $(\mathrm{kg})$ & $18.1 \pm 4.1$ & $21.6 \pm 5.2$ & $<0.001$ \\
\hline PB glucose (mg/dL) & $98 \pm 34$ & $100 \pm 24$ & 0.656 \\
\hline PB insulin $(\mu \mathrm{U} / \mathrm{mL})$ & $7.1 \pm 6.0$ & $8.9 \pm 7.8$ & 0.132 \\
\hline PB triglycerides (mg/dL) & $130 \pm 66$ & $147 \pm 86$ & 0.148 \\
\hline Cholesterol (mg/dL) & $215 \pm 32$ & $221 \pm 32$ & 0.190 \\
\hline HDL cholesterol (mg/dL) & $64 \pm 14$ & $63 \pm 15$ & 0.535 \\
\hline Leptin (ng/mL) & $6.8 \pm 4.0$ & $8.6 \pm 5.1$ & 0.008 \\
\hline Adiponectin (mg/L) & $15.5 \pm 7.6$ & $12.6 \pm 8.0$ & 0.017 \\
\hline Leucocytes $\left(10^{9} / \mathrm{L}\right)$ & $6.2 \pm 1.6$ & $6.0 \pm 1.6$ & 0.599 \\
\hline PAl-1 (ng/mL) & $23 \pm 10$ & $26 \pm 12$ & 0.074 \\
\hline hsCRP $(\mu \mathrm{g} / \mathrm{dL})$ & $79 \pm 109$ & $92 \pm 114$ & 0.466 \\
\hline TNF- $\alpha(p g / m L)$ & $1.6 \pm 0.8$ & $1.7 \pm 1.2$ & 0.615 \\
\hline Red blood cells $\left(10^{12} / \mu \mathrm{L}\right)$ & $421 \pm 34$ & $423 \pm 37$ & 0.691 \\
\hline Hemoglobin $(\mathrm{g} / \mathrm{L})$ & $127 \pm 10$ & $129 \pm 12$ & 0.367 \\
\hline Hematocrit (\%) & $40.7 \pm 3.0$ & $40.8 \pm 3.6$ & 0.838 \\
\hline Albumin (g/dL) & $4.4 \pm 0.2$ & $4.4 \pm 0.3$ & 0.209 \\
\hline Iron $(\mu \mathrm{g} / \mathrm{dL})$ & $93 \pm 27$ & $94 \pm 28$ & 0.908 \\
\hline Copper ( $\mu \mathrm{g} / \mathrm{dL})$ & $110 \pm 17$ & $109 \pm 14$ & 0.604 \\
\hline $\operatorname{Zinc}(\mu \mathrm{g} / \mathrm{dL})$ & $77 \pm 11$ & $77 \pm 11$ & 0.823 \\
\hline Transthyretin (mg/dL) & $27 \pm 5$ & $27 \pm 6$ & 0.436 \\
\hline Creatinine (mg/dL) & $0.67 \pm 0.12$ & $0.71 \pm 0.19$ & 0.080 \\
\hline eGFR $\left(\mathrm{mL} / \mathrm{min} / 1.73 \mathrm{~m}^{2}\right)$ & $66 \pm 12$ & $64 \pm 15$ & 0.540 \\
\hline Anemia (n, \%) & $19,21.8$ & $17,19.1$ & 0.709 \\
\hline CKD G3b (n, \%) & $4,4.6$ & $8,9.0$ & 0.371 \\
\hline Hyperadiponectinemia (n, \%) & $23,26.4$ & $11,12.4$ & 0.022 \\
\hline
\end{tabular}

Mean \pm SD or $n, \%$.

BMI, body mass index; CKD, chronic kidney disease; FMI, fat mass index; HDL, high-density lipoprotein; PAI-1, plasminogen activatorinhibitor-1; PB, post-breakfast; RBC, red blood cells; SMI, skeletal muscle mass index; TNF- $\alpha$, tumor necrosis factor-alpha; eGFR, estimated glomerular filtration rate; hsCRP, high-sensitivity C-reactive protein.

maintained as assessed by their mean BMI, cholesterol, serum albumin and TTR. Anemia and CKD were found in $36(20.5 \%)$ and 58 women $(33.0 \%)$, respectively. Among women with CKD, 12 women had CKD G3b.

Women with low grip strength as compared with those without low grip strength were older, of short stature, lighter build and had lower BMI, fat mass, SM and SMI (all $\mathrm{p}<0.05$ or less) (table 1 ). They had higher serum adiponectin $(\mathrm{p}=0.003)$ and higher percentage of women with hyperadiponectinemia $(\mathrm{p}=0.006)$ despite no difference in waist circumference, FMI and serum leptin. There was also no difference in PB glucose, insulin and triglycerides and HDL cholesterol. TNF- $\alpha$ was higher $(\mathrm{p}=0.025)$ in women with low grip strength 
Table 3 Anthropometric and laboratory characteristics of women with sarcopenia

\begin{tabular}{|c|c|c|c|}
\hline & \multicolumn{2}{|c|}{ Sarcopenia } & \multirow[b]{3}{*}{$P$ values } \\
\hline & Yes & No & \\
\hline & $\mathrm{n}=45$ & $n=131$ & \\
\hline Age (years) & $80.1 \pm 5.2$ & $75.6 \pm 8.7$ & $<0.01$ \\
\hline Height (cm) & $145.5 \pm 5.2$ & $149.9 \pm 5.9$ & $<0.01$ \\
\hline Weight (kg) & $43.7 \pm 6.3$ & $51.8 \pm 7.0$ & $<0.01$ \\
\hline $\operatorname{BMI}\left(\mathrm{kg} / \mathrm{m}^{2}\right)$ & $20.7 \pm 2.9$ & $23.0 \pm 2.8$ & $<0.01$ \\
\hline FMI $\left(\mathrm{kg} / \mathrm{m}^{2}\right)$ & $6.4 \pm 2.4$ & $7.7 \pm 2.3$ & 0.002 \\
\hline $\mathrm{SMI}\left(\mathrm{kg} / \mathrm{m}^{2}\right)$ & $5.1 \pm 0.4$ & $6.0 \pm 0.6$ & $<0.01$ \\
\hline Waist circumference $(\mathrm{cm})$ & $83.2 \pm 9.5$ & $87.5 \pm 8.9$ & 0.007 \\
\hline Fat mass (kg) & $13.5 \pm 5.2$ & $17.2 \pm 5.2$ & $<0.001$ \\
\hline Skeletal muscle mass $(\mathrm{kg})$ & $10.8 \pm 1.1$ & $13.4 \pm 1.9$ & $<0.001$ \\
\hline Grip strength (kg) & $14.9 \pm 2.6$ & $21.6 \pm 4.5$ & $<0.001$ \\
\hline PB glucose (mg/dL) & $99 \pm 43$ & $100 \pm 23$ & 0.828 \\
\hline PB insulin $(\mu \mathrm{U} / \mathrm{mL})$ & $7.0 \pm 6.1$ & $8.5 \pm 7.4$ & 0.327 \\
\hline PB triglycerides (mg/dL) & $128 \pm 66$ & $142 \pm 81$ & 0.254 \\
\hline Cholesterol (mg/dL) & $208 \pm 26$ & $222 \pm 33$ & 0.005 \\
\hline HDL cholesterol (mg/dL) & $64 \pm 13$ & $63 \pm 15$ & 0.664 \\
\hline Leptin (ng/mL) & $6.6 \pm 4.4$ & $8.1 \pm 4.7$ & 0.059 \\
\hline Adiponectin (mg/L) & $16.6 \pm 7.9$ & $13.2 \pm 7.8$ & 0.010 \\
\hline Leucocytes $\left(10^{9} / \mathrm{L}\right)$ & $6.2 \pm 1.6$ & $6.1 \pm 1.6$ & 0.796 \\
\hline PAl-1 (ng/mL) & $23 \pm 11$ & $25 \pm 12$ & 0.368 \\
\hline hsCRP ( $\mu \mathrm{g} / \mathrm{dL})$ & $74 \pm 84$ & $90 \pm 119$ & 0.407 \\
\hline TNF- $\alpha(p g / m L)$ & $1.7 \pm 0.8$ & $1.7 \pm 1.1$ & 0.764 \\
\hline Red blood cells $\left(10^{12} / L\right)$ & $417 \pm 39$ & $424 \pm 34$ & 0.205 \\
\hline Hemoglobin $(g / l)$ & $126 \pm 10$ & $129 \pm 11$ & 0.113 \\
\hline Hematocrit (\%) & $40.4 \pm 3.0$ & $40.9 \pm 3.4$ & 0.411 \\
\hline Albumin (g/dL) & $4.30 \pm 0.27$ & $4.41 \pm 0.25$ & 0.009 \\
\hline Iron $(\mu \mathrm{g} / \mathrm{dL})$ & $93 \pm 28$ & $94 \pm 27$ & 0.815 \\
\hline Copper $(\mu \mathrm{g} / \mathrm{dL})$ & $111 \pm 18$ & $109 \pm 14$ & 0.393 \\
\hline $\operatorname{Zinc}(\mu \mathrm{g} / \mathrm{dL})$ & $74 \pm 10$ & $78 \pm 11$ & 0.057 \\
\hline Transthyretin (mg/dL) & $26 \pm 5$ & $28 \pm 5$ & 0.049 \\
\hline Creatinine (mg/dL) & $0.66 \pm 0.13$ & $0.70 \pm 0.16$ & 0.141 \\
\hline eGFR $\left(\mathrm{mL} / \mathrm{min} / 1.73 \mathrm{~m}^{2}\right)$ & $66 \pm 13$ & $64 \pm 13$ & 0.366 \\
\hline Anemia (n, \%) & $12,27.3$ & $24,18.3$ & 0.146 \\
\hline CKD3b (n, \%) & $4,8.9$ & $8,6.1$ & 0.506 \\
\hline Hyperadiponectinemia (n, \%) & $15,33.3$ & $19,14.5$ & 0.007 \\
\hline
\end{tabular}

Mean \pm SD or $n, \%$.

BMI, body mass index; CKD, chronic kidney disease; FMI, fat mass index; HDL, high-density lipoprotein; PAI-1, plasminogen activatorinhibitor-1; PB, post-breakfast; RBC, red blood cells; SMI, skeletal muscle mass index; TNF- $\alpha$, tumor necrosis factor-alpha; eGFR, estimated glomerular filtration rate; hsCRP, high-sensitivity C-reactive protein.

as compared with those without but other markers of inflammation (leucocyte counts, hsCRP and PAI-1) did not differ. Although women with low grip strength had lower hemoglobin $(p=0.004)$, the percentage of women with anemia did not differ between the two groups. Serum albumin, TTR (both $\mathrm{p}<0.001)$ and zinc $(\mathrm{p}=0.005)$ were lower in women with low grip strength compared with those without low grip strength. There was also no group difference in mean eGFR and the percentage of CKD G3b (table 1).

Women with low muscle mass as compared with those without low muscle mass were older $(\mathrm{p}=0.006)$, of short stature $(\mathrm{p}=0.008)$ and lighter build and had lower anthropometric indices (all $\mathrm{p}<0.001)$ studied and serum leptin 
Table 4 Logistic regression analyses for low grip strength

OR $95 \% \mathrm{Cl} \quad$ P values

\begin{tabular}{|lllc}
\hline (A) Bivariate analyses & & & \\
\hline Age & 1.14 & 1.091 to 1.221 & $<0.001$ \\
\hline Height & 0.80 & 0.752 to 0.872 & $<0.001$ \\
\hline Weight & 0.80 & 0.835 to 0.927 & $<0.001$ \\
\hline Fat mass & 0.93 & 0.859 to 0.970 & $<0.001$ \\
\hline Skeletal muscle mass & 0.61 & 0.528 to 0.779 & 0.001 \\
\hline Log TNF- $\alpha$ & 4.81 & 1.187 to 19.413 & 0.005 \\
\hline Hemoglobin & 0.62 & 0.485 to 0.878 & $<0.001$ \\
\hline Albumin & 0.08 & 0.018 to 0.263 & $<0.001$ \\
\hline TTR & 0.85 & 0.827 to 0.948 & 0.007 \\
\hline Zinc & 0.99 & 0.931 to 0.988 & 0.006 \\
\hline Hyperadiponectinemia & 2.92 & 1.373 to 6.437 & 0.011 \\
\hline (B) Multivariate analyses & & & $<0.001$ \\
\hline Age & 1.03 & 1.021 to 1.171 & 0.018 \\
\hline Height & 0.88 & 0.779 to 0.923 & 0.017 \\
\hline Skeletal muscle mass & 0.72 & 0.594 to 0.951 & 0.003 \\
\hline TTR & 0.92 & 0.829 to 0.982 & $<0.001$ \\
\hline (C) Multivariate analyses & & & 0.032 \\
\hline Age & 1.16 & 1.034 to 1.183 & \\
\hline Height & 0.81 & 0.783 to 0.926 & 0.621 to 0.979 \\
\hline Skeletal muscle mass & 0.70 & & \\
\hline
\end{tabular}

Both $A$ and $B$ included all variables that showed significant differences between women with and without low grip strength in table 1; age, body weight, height, fat and skeletal muscle mass, TNF- $\alpha$, hemoglobin, albumin, TTR, zinc and hyperadiponectinemia. In C, TTR was removed from $B$.

BMI, body mass index; CKD, chronic kidney disease; FMI, fat mass index; HDL, high-density lipoprotein; PAI-1, plasminogen activatorinhibitor-1; PB, post-breakfast; RBC, red blood cells.; SMI, skeletal muscle mass index; TNF- $\alpha$, tumor necrosis factor-alpha; TTR, transthyretin; eGFR, estimated glomerular filtration rate; hsCRP, high-sensitivity C-reactive protein.

$(\mathrm{p}=0.008)$ (table 2$)$. They had higher serum adiponectin $(\mathrm{p}=0.017)$ and higher percentage of women with hyperadiponectinemia $(\mathrm{p}=0.022)$ as well. Other variables studied did not differ between the two groups of women.

Sarcopenia was associated older age, short stature, lighter build and lower anthropometric indices studied (all $\mathrm{p} \leq 0.001$ ) (table 3). Sarcopenic women had higher percentage of women with hyperadiponectinemia $(\mathrm{p}=0.007)$ and higher serum adiponectin $(\mathrm{p}=0.010)$, and lower serum cholesterol $(\mathrm{p}=0.005)$, albumin $(\mathrm{p}=0.009)$ and TTR $(\mathrm{p}=0.049)$. Serum leptin and zinc tended to be lower (both $\mathrm{p}=0.05$ ). Other variables studied did not significantly differ between the two groups of women in the presence and absence of sarcopenia.

Bivariate logistic regression analyses were done between low grip strength and all variables that showed significant differences between women with and without low grip strength in table 1 , except for serum adiponectin concentrations (table 4, (A); age, body weight, height, fat and skeletal muscle mass, TNF- $\alpha$, hemoglobin, albumin, TTR, zinc and hyperadiponectinemia. Low grip strength was associated with all these variables. In multivariable logistic regression analysis, which included all variables in $\mathrm{A}$ as independent variables (table 4, (B), age, height, skeletal muscle mass and TTR but not hyperadiponectinemia emerged as independent determinants of low grip strength. When TTR was removed (C), hyperadiponectinemia did not emerge as an independent determinant of low grip strength.

Serum adiponectin concentrations were associated with low grip strength as well (OR 1.07; 95\% CI 1.02 to 1.12, $\mathrm{p}=0.002$ ). Multivariate logistic regression analysis yielded almost the same results when serum adiponectin instead of hyperadiponectinemia was included as independent variables (data not shown).

In bivariate logistic regression analyses, low SMI and sarcopenia were associated with variables that showed significant differences in tables 2 and 3, respectively (data not shown). However, in multivariate logistic regression analyses, hyperadiponectinemia did not emerge as an independent determinant of low SMI or sarcopenia.

Among 129 women who did not have sarcopenia, 63 women had normal muscle mass and grip strength and 66 had either low SM $(n=42)$ or low grip strength alone $(\mathrm{n}=26)$ (termed as pre-sarcopenia) (online supplementary table 1). Compared with women with normal muscle 
mass and grip strength, pre-sarcopenic and sarcopenic women were older and had lower height and fat mass and higher serum adiponectin and percentages of women with hyperadiponectinemia. Weight, SM, blood hemoglobin, serum albumin and TTR decreased in a stepwise fashion from normal SMI and grip strength through pre-sarcopenia to sarcopenia.
Hyperadiponectinemia was associated with older age, smaller body size, lower grip strength and SMI, and higher percentage of low grip strength, low SMI and sarcopenia (all $\mathrm{p}<0.01$ or less) (table 5 ). It was also associated with lower waist circumference, insulin, triglycerides and leptin, and higher HDL cholesterol (all $\mathrm{p}<0.001$ ). In addition, hyperadiponectinemia was associated with

Table 5 Features of elderly women with hyperadiponectinemia

\begin{tabular}{|c|c|c|c|}
\hline & Hyperadip & & \\
\hline & Yes & No & \\
\hline & $\mathrm{n}=34$ & $n=142$ & $P$ values \\
\hline Age (years) & $80.1 \pm 7.2$ & $75.9 \pm 8.2$ & 0.007 \\
\hline Height (cm) & $146.3 \pm 6.7$ & $149.4 \pm 5.7$ & 0.007 \\
\hline Weight (kg) & $43.7 \pm 6.4$ & $51.2 \pm 7.3$ & $<0.001$ \\
\hline $\operatorname{BMI}\left(\mathrm{kg} / \mathrm{m}^{2}\right)$ & $20.4 \pm 2.9$ & $22.9 \pm 2.8$ & $<0.001$ \\
\hline FMI $\left(\mathrm{kg} / \mathrm{m}^{2}\right)$ & $5.6 \pm 2.1$ & $7.8 \pm 2.2$ & $<0.001$ \\
\hline $\mathrm{SMI}\left(\mathrm{kg} / \mathrm{m}^{2}\right)$ & $5.5 \pm 0.6$ & $5.8 \pm 0.7$ & 0.007 \\
\hline Waist circumference $(\mathrm{cm})$ & $80.7 \pm 8.9$ & $87.8 \pm 8.8$ & $<0.001$ \\
\hline Fat mass (kg) & $11.8 \pm 4.5$ & $17.3 \pm 5.1$ & $<0.001$ \\
\hline Skeletal muscle mass (kg) & $11.7 \pm 1.8$ & $13.0 \pm 2.1$ & 0.001 \\
\hline Grip strength (kg) & $17.4 \pm 4.6$ & $20.5 \pm 4.9$ & 0.002 \\
\hline Low grip strength (n, \%) & $21,61.8$ & $50,35.2$ & 0.006 \\
\hline Low SMI (n, \%) & $23,67.6$ & $64,45.1$ & 0.023 \\
\hline Sarcopenia (n, \%) & $15,44.1$ & $30,21.1$ & 0.011 \\
\hline PB glucose (mg/dL) & $92 \pm 17$ & $101 \pm 32$ & 0.109 \\
\hline PB insulin $(\mu \mathrm{U} / \mathrm{mL})$ & $4.9 \pm 4.2$ & $8.8 \pm 7.4$ & 0.001 \\
\hline PB triglycerides (mg/dL) & $96 \pm 43$ & $149 \pm 80$ & $<0.001$ \\
\hline Cholesterol (mg/dL) & $215 \pm 29$ & $219 \pm 33$ & 0.586 \\
\hline HDL cholesterol (mg/dL) & $72 \pm 15$ & $61 \pm 13$ & $<0.001$ \\
\hline Leptin (ng/mL) & $4.9 \pm 3.0$ & $8.4 \pm 4.8$ & $<0.001$ \\
\hline Adiponectin (mg/L) & $26.6 \pm 7.2$ & $11.0 \pm 4.3$ & $<0.001$ \\
\hline Leucocytes (10\%/L) & $5.3 \pm 1.4$ & $6.3 \pm 1.6$ & 0.001 \\
\hline hsCRP ( $\mu \mathrm{g} / \mathrm{dL})$ & $66 \pm 88$ & $90 \pm 116$ & 0.257 \\
\hline TNF- $\alpha(p g / m L)$ & $1.6 \pm 0.7$ & $1.7 \pm 1.1$ & 0.570 \\
\hline Red blood cells $\left(10^{12} / L\right)$ & $399 \pm 38$ & $428 \pm 33$ & $<0.001$ \\
\hline Hemoglobin (g/L) & $122 \pm 11$ & $130 \pm 10$ & 0.001 \\
\hline Hematocrit (\%) & $39.0 \pm 3.4$ & $41.2 \pm 3.2$ & $<0.001$ \\
\hline Albumin (g/dL) & $4.3 \pm 0.3$ & $4.4 \pm 0.2$ & 0.184 \\
\hline Iron $(\mu \mathrm{g} / \mathrm{dL})$ & $90 \pm 28$ & $94 \pm 27$ & 0.440 \\
\hline Copper $(\mu \mathrm{g} / \mathrm{dL})$ & $110 \pm 16$ & $109 \pm 15$ & 0.876 \\
\hline $\operatorname{Zinc}(\mu \mathrm{g} / \mathrm{dL})$ & $77 \pm 12$ & $77 \pm 11$ & 0.916 \\
\hline Transthyretin (mg/dL) & $26 \pm 4$ & $27 \pm 5$ & 0.049 \\
\hline Creatinine (mg/dL) & $0.70 \pm 0.19$ & $0.68 \pm 0.15$ & 0.567 \\
\hline eGFR $\left(\mathrm{mL} / \mathrm{min} / 1.73 \mathrm{~m}^{2}\right)$ & $64 \pm 14$ & $65 \pm 13$ & 0.600 \\
\hline Anemia (n, \%) & 1338.2 & 2316.2 & 0.008 \\
\hline CKD G3b (n, \%) & 411.8 & 85.6 & 0.250 \\
\hline
\end{tabular}

Mean \pm SD or $n, \%$.

BMI, body mass index; CKD, chronic kidney disease; FMI, fat mass index; HDL, high-density lipoprotein; PAI-1, plasminogen activator-inhibitor-1; PB, post-breakfast; RBC, red blood cells; SMI, skeletal muscle mass index; TNF- $\alpha$, tumor necrosis factor-alpha; eGFR, estimated glomerular filtration rate; hsCRP, high-sensitivity C-reactive protein. 
lower leucocyte counts, hemoglobin (both $\mathrm{p}=0.001$ ) and TTR $(p=0.049)$, and higher percentage of anemia $(\mathrm{p}=0.008)$. However, there was no association with hsCRP, $\mathrm{TNF}-\alpha$ and kidney function.

\section{DISCUSSION}

Present results in Japanese elderly women living in local community have demonstrated that low grip strength was associated not only with nutrition status and inflammation but also with elevated adiponectin, whereas low muscle mass was associated with hyperadiponectinemia alone. Although sarcopenia was associated with hyperadiponectinemia and nutrition status, hyperadiponectinemia was not an independent determinant of low muscle strength and low muscle mass, either alone or in combination. We confirmed previous studies as described later. ${ }^{13-16} 26$ It is noteworthy that our elderly women appeared to have preserved insulin sensitivity as mean values of waist circumference, BMI and HDL cholesterol were within the respective normal range of the cut-off value for the diagnosis of the metabolic syndrome and obesity for Japanese (waist $>90 \mathrm{~cm}$, BMI $>25.0 \mathrm{~kg} / \mathrm{m}^{2}$, HDL cholesterol $<50 \mathrm{mg} / \mathrm{dL}) .{ }^{27}{ }^{28}$ Further, these findings were observed in community-living elderly Japanese women who had fewer indicators of disease, such as a low BMI, hypoalbuminemia or hypocholesterolemia.

In seriously ill patients, very low TTR concentrations are inversely related to CRP and reflect severity of illness rather than nutritional status. ${ }^{29}$ In patient who requires careful assessment and monitoring for nutritional status, TTR is helpful for identifying at-risk patients. ${ }^{30}$ Because we did not evaluate food intake in the present study, subtle alterations in energy-to-protein balance may be related to low TTR. ${ }^{31}$ In elderly Japanese women with mean CRP of $0.085 \mathrm{mg} / \mathrm{dL}$, we have previously shown that TTR was independently associated with hsCRP, suggesting that TTR may be a very sensitive marker of systemic low-grade inflammation. ${ }^{19}$ Whether decreased serum TTR is related more to nutrition or to disease remains controversial and, especially among older people, it is difficult to distinguish the effects of undernutrition from those of disease. In the present study, low TTR was associated with low grip strength independently of hsCRP and other confounding factors, suggesting that TTR is unlikely to be a marker of low-grade inflammation in this situation. Finally, circulating TTR may be a biomarker of age-related catabolic states. ${ }^{31}$

The possible association between inflammatory parameters and sarcopenia is controversial and poorly understood. A recent meta-analysis reported that sarcopenia seems to be associated with elevated serum CRP. ${ }^{32}$ In contrast, cohort studies have indicated TNF- $\alpha$ and interleukin-6 levels as markers of sarcopenia or frailty. ${ }^{10} 33$ The latter observations may be in line with our findings that TNF- $\alpha$, but not hsCRP, was associated with low grip strength in elderly Japanese women. This may be related to low hsCRP with a mean value of $0.083 \mathrm{mg} / \mathrm{dL}$ in women with low grip strength in the present study.

We have previously reported elevated adiponectin in elderly women with anemia and CKD G3b. ${ }^{17}$ However, there was no difference in the percentage of women with anemia and CKD G3b between women with and without low grip strength, low SMI and sarcopenia in the present study. We have previously shown in elderly women that the prevalence of CKD was much higher when creatinine-based eGFR was used than the prevalence obtained when cystatin-C based equations were used ( $46 \%$ vs $13 \%$, $\mathrm{p}<0.001) .{ }^{34}$ This may explain in part why percentage of women with CKD was high (33\%) in the present study.

Biology underlying inverse association between adiponectin and grip strength or muscle mass remains unclear. Adiponectin in the circulation may serve a general 'housekeeping' function by facilitating phagocytosis of apoptotic cells by macrophages. ${ }^{35}$ This anti-inflammatory property would make hyperadiponectinemia in response to underlying disease a marker of illness severity and worse prognosis. Another explanation is muscle adiponectin resistance, which has been demonstrated in patients with heart failure, characterized by skeletal muscle weakness and wasting. ${ }^{36}$ Adiponectin expression has been shown to be increased in skeletal muscle in patients with heart failure, with concurrent downregulation of adiponectin receptor and the receptor's downstream pathways, indicative of adiponectin resistance. ${ }^{36}$ These findings raise the possibility that ageing-associated muscle weakness and muscle loss might exhibit the same pattern of adiponectin expression and local resistance as observed in skeletal muscle wasting in chronic heart failure. If this has been demonstrated to be the case, adiponectin resistance may precede IR in Japanese elderly women with low grip strength and muscle mass, either alone or in combination, as their insulin sensitivity appeared to be preserved as described above. An inverse association between adiponectin and grip strength and muscle mass may be compatible with a recent experimental study that the administration of AdipoRon, an agonist for adiponectin receptors, had negative impact on muscle mass in myotubes and mouse skeletal muscle. ${ }^{37}$

We have previously reported a positive association between blood hemoglobin levels and grip strength. ${ }^{18}$ In the present study, however, association between low grip strength and hemoglobin was not significant in a fully adjusted model, although it was significant in unadjusted analysis. Blood hemoglobin also has been shown to be positively associated with IR. ${ }^{38}$ Therefore, lower hemoglobin would be associated with an increase in insulin sensitivity in women with low grip strength in the present study.

Cross-sectional associations between elevated serum adiponectin and low muscle mass, muscle strength and poor physical functioning have been shown in adult employees, ${ }^{15}$ young and old healthy subjects ${ }^{16}$ and non-cachectic, non-diabetic, elderly patients with chronic heart 
failure. ${ }^{39}$ Prospective studies have demonstrated that higher adiponectin was associated with muscle strength and poor physical functioning in middle-aged and the elderly people. ${ }^{14152640}$ An increase in adiponectin levels over time has been reported to be associated with greater physical disability in long-lived adults. ${ }^{13}$

Several limitations must be acknowledged. The cross-sectional design did not allow causal relationship. The recruitment procedure may also have some potential impact on the results. As the participation was voluntary, women who pay more attention to health may be more likely to participate. Laboratory parameters were measured only once and time interval between breakfast and blood samplings varied. This likely has some influence on parameters measured, for example, adiponectin. ${ }^{41}$ It is reported that serum adiponectin reached a nadir at night and was rising in the early morning. ${ }^{41}$ However, no difference was reported in postprandial adiponectin changes between a high fat versus an isoenergetic low-fat meal $^{42}$ and between lean and obese non-diabetic men. ${ }^{43}$ We did not have detailed drug information. Some antihypertensive medications may have effects on muscle mass ${ }^{44}$ and both thiazolidinediones and renin-angiotensin system inhibitors had effects on adiponectin levels. ${ }^{45} 46$ As we studied Japanese elderly women only, results may not be generalized to other races or ethnicities. Further, we used many surrogate markers which would not be accurate. Finally, we did not measure plasma brain natriuretic peptide, the strongest predictor of circulating adiponectin. ${ }^{47}$

In conclusion, low grip strength and low muscle mass, either alone or in combination (sarcopenia), were associated with elevated serum adiponectin in community-living Japanese elderly women with preserved IR. Low grip strength, but not low muscle mass, was associated with decreased TTR, a marker of nutrition and age-related catabolic states as well. The inverse association of adiponectin with muscle strength and mass suggests an opportunity to identify those at high risk of this complication and to discover insights into the mechanisms of this very early adverse event.

\section{Author affiliations}

${ }^{1}$ Department of Food Sciences and Nutrition, School of Human Environmental Sciences, Mukogawa Women's University, Nishinomiya, , Japan

${ }^{2}$ Research Institute for Nutrition Sciences, Mukogawa Women's University, Nishinomiya, Japan

${ }^{3}$ Department of Nutrition, Osaka City Juso Hospital, Osaka, Japan

${ }^{4}$ Graduate School of Human Science and Environment, University of Hyogo, Himeji, Japan

${ }^{5}$ Department of Nutritional Sciences for Well-being, Faculty of Health Sciences for Welfare, Kansai University of Welfare Sciences, Kashiwara, Osaka, Japan ${ }^{6}$ Diabetes Division, Department of Medicine, Kohnan Kakogawa Hospital, Kakogawa, Japan

Acknowledgements We are indebted to all the participants for their dedicated and conscientious collaboration.

Contributors MT, AT, SM, MY, KK and MK collected and analyzed data. TK wrote the manuscript and KF reviewed and edited it. All authors approved the final version of the manuscript to be published. TK supervised the study, had full access to all the data in the study and takes responsibility for the integrity of the data and the accuracy of the data analysis.

Funding The authors have not declared a specific grant for this research from any funding agency in the public, commercial or not-for-profit sectors.

Competing interests None declared.

Patient consent for publication Not required.

Ethics approval The Ethics Committees of the Mukogawa Women's University (No. $11-7$ on $20 / 5 / 2011)$.

Provenance and peer review Not commissioned; externally peer reviewed.

Data availability statement № data are available.

Open access This is an open access article distributed in accordance with the Creative Commons Attribution Non Commercial (CC BY-NC 4.0) license, which permits others to distribute, remix, adapt, build upon this work non-commercially, and license their derivative works on different terms, provided the original work is properly cited, appropriate credit is given, any changes made indicated, and the use is non-commercial. See: http://creativecommons.org/licenses/by-nc/4.0/.

\section{REFERENCES}

1. Cruz-Jentoft AJ, Baeyens JP, Bauer JM, et al. Sarcopenia: European consensus on definition and diagnosis: report of the European Working group on sarcopenia in older people. Age Ageing 2010;39:412-23.

2. Tieland M, Trouwborst I, Clark BC. Skeletal muscle performance and ageing. J Cachexia Sarcopenia Muscle 2018;9:3-19.

3. Abbatecola AM, Ferrucci L, Ceda G, et al. Insulin resistance and muscle strength in older persons. J Gerontol A Biol Sci Med Sci 2005;60:1278-82.

4. Barzilay JI, Cotsonis GA, Walston J, et al. Insulin resistance is associated with decreased quadriceps muscle strength in nondiabetic adults aged >=70 years. Diabetes Care 2009;32:736-8.

5. Lazarus R, Sparrow D, Weiss ST. Handgrip strength and insulin levels: cross-sectional and prospective associations in the normative aging study. Metabolism 1997;46:1266-9.

6. Li JJ, Wittert GA, Vincent A, et al. Muscle grip strength predicts incident type 2 diabetes: population-based cohort study. Metabolism 2016;65:883-92.

7. Wander PL, Boyko EJ, Leonetti DL, et al. Greater hand-grip strength predicts a lower risk of developing type 2 diabetes over 10 years in leaner Japanese Americans. Diabetes Res Clin Pract 2011;92:261-4.

8. Marques-Vidal P, Vollenweider P, Waeber G, et al. Grip strength is not associated with incident type 2 diabetes mellitus in healthy adults: the CoLaus study. Diabetes Res Clin Pract 2017;132:144-8.

9. Reid MB, Li Y-P. Tumor necrosis factor-alpha and muscle wasting: a cellular perspective. Respir Res 2001;2:269-72.

10. Beyer I, Mets T, Bautmans I. Chronic low-grade inflammation and age-related sarcopenia. Curr Opin Clin Nutr Metab Care 2012;15:12-22.

11. Modan-Moses D, Stein D, Pariente C, et al. Modulation of adiponectin and leptin during refeeding of female anorexia nervosa patients. J Clin Endocrinol Metab 2007;92:1843-7.

12. Saito K, Arata S, Hosono T, et al. Adiponectin plays an important role in efficient energy usage under energy shortage. Biochim Biophys Acta 2006;1761:709-16.

13. Kizer JR, Arnold AM, Strotmeyer ES, et al. Change in circulating adiponectin in advanced old age: determinants and impact on physical function and mortality. the cardiovascular health study all stars study. J Gerontol A Biol Sci Med Sci 2010;65A:1208-14.

14. Karvonen-Gutierrez $\mathrm{CA}$, Zheng $\mathrm{H}$, Mancuso $\mathrm{P}$, et al. Higher leptin and adiponectin concentrations predict poorer performance-based physical functioning in midlife women: the Michigan Study of Women's Health across the nation. J Gerontol A Biol Sci Med Sci 2016;71:508-14.

15. Huang $\mathrm{C}$, Niu K, Momma H, et al. Inverse association between circulating adiponectin levels and skeletal muscle strength in Japanese men and women. Nutr Metab Cardiovasc Dis 2014;24:42-9.

16. Bucci L, Yani SL, Fabbri C, et al. Circulating levels of adipokines and IGF-1 are associated with skeletal muscle strength of young and old healthy subjects. Biogerontology 2013;14:261-72.

17. Tsuboi A, Watanabe M, Kazumi T, et al. Anemia and reduced renal function are independent predictors of elevated serum adiponectin in elderly women. J Atheroscler Thromb 2013;20:568-74.

18. Yamada E, Takeuchi M, Kurata M, et al. Low haemoglobin levels contribute to low grip strength independent of low-grade 
inflammation in Japanese elderly women. Asia Pac J Clin Nutr 2015;24:444-51.

19. Tsuboi A, Terazawa-Watanabe M, Kazumi T, et al. Associations of decreased serum transthyretin with elevated high-sensitivity CRP, serum copper and decreased hemoglobin in ambulatory elderly women. Asia Pac J Clin Nutr 2015;24:83-9.

20. Chen L-K, Liu L-K, Woo J, et al. Sarcopenia in Asia: consensus report of the Asian Working group for sarcopenia. J Am Med Dir Assoc 2014;15:95-101.

21. Tanaka S, Wu B, Honda M, et al. Associations of lower-body fat mass with favorable profile of lipoproteins and adipokines in healthy, slim women in early adulthood. J Atheroscler Thromb 2011;18:365-72.

22. Tsuboi A, Watanabe M, Kazumi T, et al. Association of low serum iron levels with low-grade inflammation and hyperadiponectinemia in community-living elderly women. $J$ Atheroscler Thromb 2013;20:670-7.

23. Rambod M, Kovesdy CP, Bross R, et al. Association of serum prealbumin and its changes over time with clinical outcomes and survival in patients receiving hemodialysis. Am J Clin Nutr 2008;88:1485-94.10.3945/ajcn.2008.2590610.3945/ ajcn.2008.25906

24. Matsuo S, Imai E, Horio M, et al. Revised equations for estimated GFR from serum creatinine in Japan. Am J Kidney Dis 2009;53:982-92.

25. Levey AS, de Jong PE, Coresh J, et al. The definition, classification, and prognosis of chronic kidney disease: a KDIGO controversies conference report. Kidney Int 2011;80:17-28.

26. Baker JF, Newman AB, Kanaya A, et al. The adiponectin paradox in the elderly: associations with body composition, physical functioning, and mortality. J Gerontol A Biol Sci Med Sci 2019;74:247-53

27. Alberti KGMM, Eckel RH, Grundy SM, et al. Harmonizing the metabolic syndrome: a joint interim statement of the International diabetes Federation Task force on epidemiology and prevention; National heart, lung, and blood Institute; American heart association; world heart Federation; international atherosclerosis Society; and international association for the study of obesity. Circulation 2009;120:1640-5.

28. Examination Committee of Criteria for 'Obesity Disease' in Japan, Japan Society for the Study of Obesity. New criteria for 'obesity disease' in Japan. Circ J 2002;66:987-92.

29. Clark MA, Hentzen BTH, Plank LD, et al. Sequential changes in insulin-like growth factor 1 , plasma proteins, and total body protein in severe sepsis and multiple injury. JPEN J Parenter Enteral Nutr 1996;20:363-70.

30. Shenkin A. Serum prealbumin: is it a marker of nutritional status or of risk of malnutrition? Clin Chem 2006;52:2177-9.

31. Ingenbleek $Y$, Bernstein LH. Plasma transthyretin as a biomarker of lean body mass and catabolic states. Adv Nutr 2015;6:572-80.

32. Bano G, Trevisan C, Carraro S, et al. Inflammation and sarcopenia: a systematic review and meta-analysis. Maturitas 2017;96:10-15.
33. Michaud M, Balardy L, Moulis G, et al. Proinflammatory cytokines, aging, and age-related diseases. J Am Med Dir Assoc 2013;14:877-82.

34. Kurata M, Tsuboi A, Takeuchi M, et al. Association of metabolic syndrome with chronic kidney disease in elderly Japanese women: comparison by estimation of glomerular filtration rate from creatinine, cystatin C, and both. Metab Syndr Relat Disord 2016;14:40-5.

35. Ouchi N, Walsh $\mathrm{K}$. A novel role for adiponectin in the regulation of inflammation. Arterioscler Thromb Vasc Biol 2008;28:1219-21.

36. Sente T, Van Berendoncks AM, Hoymans VY, et al. Adiponectin resistance in skeletal muscle: pathophysiological implications in chronic heart failure. J Cachexia Sarcopenia Muscle 2016;7:261-74.

37. Ito R, Higa M, Goto A, et al. Activation of adiponectin receptors has negative impact on muscle mass in $\mathrm{C} 2 \mathrm{C} 12$ myotubes and fast-type mouse skeletal muscle. PLoS One 2018;13:e0205645.10.1371/ journal.pone.020564510.1371/journal.pone.0205645

38. Hanley AJG, Retnakaran R, Qi Y, et al. Association of hematological parameters with insulin resistance and beta-cell dysfunction in nondiabetic subjects. J Clin Endocrinol Metab 2009;94:3824-32.

39. Loncar G, Bozic B, von Haehling S, et al. Association of adiponectin with peripheral muscle status in elderly patients with heart failure. Eur J Intern Med 2013;24:818-23.

40. Huang $\mathrm{C}$, Tomata $\mathrm{Y}$, Kakizaki M, et al. High circulating adiponectin levels predict decreased muscle strength among older adults aged 70 years and over: a prospective cohort study. Nutr Metab Cardiovasc Dis 2015;25:594-601.

41. Gavrila A, Peng C-K, Chan JL, et al. Diurnal and ultradian dynamics of serum adiponectin in healthy men: comparison with leptin, circulating soluble leptin receptor, and cortisol patterns. $J$ Clin Endocrinol Metab 2003;88:2838-43.

42. Kennedy A, Spiers JP, Crowley V, et al. Postprandial adiponectin and gelatinase response to a high-fat versus an isoenergetic low-fat meal in lean, healthy men. Nutrition 2015;31:863-70.

43. Phillips LK, Peake JM, Zhang X, et al. Postprandial total and HMW adiponectin following a high-fat meal in lean, obese and diabetic men. Eur J Clin Nutr 2013;67:377-84.

44. Di Bari M, Van De Poll-Franse LV, Onder G, et al. Antihypertensive medications and differences in muscle mass in older persons: the health, aging and body composition study. J Am Geriatr Soc 2004;52:961-6.

45. Riera-Guardia N, Rothenbacher D. The effect of thiazolidinediones on adiponectin serum level: a meta-analysis. Diabetes Obes Metab 2008; 10:367-75.

46. Khan BV. The effect of amlodipine besylate, losartan potassium, olmesartan medoxomil, and other antihypertensives on central aortic blood pressure and biomarkers of vascular function. Ther Adv Cardiovasc Dis 2011:5:241-73.

47. Antonopoulos AS, Margaritis M, Coutinho P, et al. Reciprocal effects of systemic inflammation and brain natriuretic peptide on adiponectin biosynthesis in adipose tissue of patients with ischemic heart disease. Arterioscler Thromb Vasc Biol 2014;34:2151-9. 